\title{
ON AUTHENTICATION, DIGITAL SIGNATURES AND SIGNATURE LAWS
}

\author{
Per Kaijser \\ SIEMENS $A G$ \\ D-81730 Munich, Germany \\ per.kaijser@mchp.siemens.de
}

\begin{abstract}
The public debate arising from the introduction of signature laws in North America, Europe and elsewhere has prompted this paper. This paper analyses and compares the closely interrelated concepts of authentication, digital signatures and electronic signature laws and tries to explain and resolve some of the ambiguities. Non-trivial problems and issues not much seen in the debate are discussed together with some suggestions as to how to overcome them. The aim of the paper is to help the promotion of both digital signatures and electronic signatures in order to improve the trust and confidence in, and acceptability of, documents in electronic form.
\end{abstract}

Keywords Authentication, digital signature, electronic signature, signature law

\section{INTRODUCTION}

Both private and public sectors are promoting transition to the information society. The great interest in this evolution is that society expects to save both work and money and at the same time create new jobs through new business opportunities. What slows down this process is a lack of trust and confidence in the new technology. Can you be confident that you are actually communicating with the right partner; can you be confident that sensitive information does not get 
into the wrong hands; can you be sure that information has not been changed during communications, and is an electronic version of a document valid as evidence in case of a dispute? These are all issues where the new technology needs to be proven acceptable and trustworthy before becoming an integral part of our society.

Trustworthy verifications of the identities of communicating partners and of sources of data are some of the necessary security features that must be supported in order to achieve confidence in the information society. The only practicable and sensible authentication method to use in an open electronic environment, where no previous knowledge of identities is assumed, is one based on public key cryptography. A digital signature is a mechanism based on this technology, which is especially well suited to this type of authentication.

There is also a need to be able to sign a document electronically with the intention of endorsing its content similar in effect to the signing of a conventional document with a hand-written signature. Several nations have introduced laws or initiated legislation in order to clarify the legal validity of electronic signatures. The intention is to give a signature in electronic form the same legal status as a hand-written signature and thereby promote the use of modern information technology. Once again a digital signature is the obvious mechanism to use.

The transition to the information society, when fully exploited, will be a drastic paradigm shift in our society. Many aspects and situations are hard to foresee and one can imagine that the transition will not be without friction, mistakes and problems. The purpose of this paper is to clarify how digital signatures, authentication, and laws dealing with the electronic equivalent to hand-written signatures are related and to discuss some specific relevant issues. It does not attempt to address all complex matters needed for a successful transition. 


\section{SOME BASIC CONCEPTS}

A Digital Signature is a well established name of a mechanism that allows a recipient of some data to prove the source and integrity of the data and protect against forgery, e.g. by the recipient [1]. It is based on an asymmetric key cryptographic algorithm and optionally a hash function. The hash function is used to transform a large piece of data into a condensed unique' value, effectively making it impossible to find another piece of data to generate the same unique' value. The asymmetric algorithm, also called a public key algorithm, makes use of a key pair, a private key and a public key. The creation of the digital signature depends on the data or, when a hash function is used, the hash value of the data to be signed and on the private key. The corresponding public key is used to verify that the signature has made use of that private key and signed the same piece of data. If the key is kept secret and if it is known who had access to that private key, one can confidently deduce who signed that piece of data.

Electronic Signature is a fairly new term that is not yet well established. It has been used in the European Union, in particular in the proposed electronic signature directive [2]. The way in which the term was used there and the way in which it will be used in this paper is to denote the electronic equivalent to a hand-written signature, which not only represents the physical signature but also protects the integrity of the content and includes the endorsement of the document content. It is thus not a mechanism, it is a function. It should, however, be pointed out that an electronic signature can never possess the same set of properties as a hand-written signature on a physical document. For example, in the electronic world one cannot distinguish between an electronic copy and an original electronic document. What they have in common, however, is that both bind the content to a source, i.e. the signer, and ensure that the content is the same as that of the original document.

A digital signature is a mechanism by which the function of an electronic signature can be achieved and verified. A digital signature can also be used for purposes other than the electronic equivalent of a hand-written signature. The word 'signature' in the name of the digital 
signature mechanism is very unfortunate and has caused much confusion. The European Commission has in its proposed directive helpfully introduced the term electronic signature, which might substantially contribute to the understanding of the difference between the function 'electronic signature' and the mechanism 'digital signature'.

An electronic signature can in principle be effected by the use of different mechanisms. The digital signature mechanism is, however, special, since it has a unique non-repudiable property, from which it follows that digital signatures can be used as proof for a third party, e.g. in a legal dispute. The field of applications of digital signatures and the application of an electronic signature can thus be viewed as two abstract areas which overlap only where the electronic signature is created by means of a digital signature.

The most essential components of a Public Key Infrastructure (PKI) are the certification authorities (CA) [3]. A CA acts as a trusted intermediary in the digital signature authentication process between the entity verifying the signature and the one creating the signature. A CA is thus a special type of trusted third party (TTP). An entity can either trust the CA directly or via a chain of trusted CAs. One of the roles of a CA is to act as the authority that states to which entity a particular public key pair belongs, i.e. the (only) entity able to use the secret private key. This ownership is normally effected by means of a public key certificate as defined in X.509 [4]. The certainty with which an entity can verify the identity of another entity relies on:

- the level of trust in the CA, i.e. mainly that its operation and personnel are trustworthy, but also that it has in place adequate physical and logical protection measures,

- measures in place to protect entities from disclosing the private keys in their possession and/or how to access the private keys,

- the cryptographic technologies, i.e. that the hash function and the public key algorithm and key size, are trustworthy, and finally

- confidence in the software and hardware application implementations and in the different underlying operating systems used by the signer, verifier and CA. 
An entity can either be a human being, an organization, or a physical entity. A physical entity is either a hardware component or a piece of software.

The total trust in the binding of a digital signature to an entity is never stronger than the weakest of the above.

\section{TYPES OF AUTHENTICATION}

Authentication is the process to prove and verify the authenticity of an identity or the authenticity of some data. There are several reasons for needing to verify the authenticity of an identity and the authenticity of data, and there are a number of authentication mechanisms. The three main reasons for performing an authentication are to verify the identity of the other entity in a communication, to verify the source and correctness of some data, and finally to verify that some data have been endorsed by an individual, i.e. that the electronic signature, which is the electronic equivalent of a hand-written signature, is authentic. These three reasons for authentication can be differentiated by calling the first one an entity or a peer authentication, the second one data authentication, and the third one electronic signature authentication.

\subsection{Entity or peer authentication}

One aspect of secure communications is to obtain assurance of the identity of the other partner of an electronic communication. Has one reached the intended recipient and where is the incoming communication really coming from? An entity authentication, or peer authentication, is the process to verify the identity of the other partner. The different processes include the verification of the identity of:

- a human being, or organization, to another human being, as in electronic commerce,

- a physical entity to another physical entity, as used in some secure communication protocols,

- a human being to a physical entity, as in a log on process, 
- an organization to a physical entity, as required in some access control processes,

- a physical entity to a human being, as in protocols that allow a user to verify that the communication is made with the right entity and not with another entity masquerading as the intended one.

The last three processes require that the identities of the verified entity are known to the verifier before the authentication process is initiated. On the other hand, the first two processes may apply to situations where the communication partners are completely unknown to each other. For instance in electronic commerce, a consumer of some goods or services is normally not known to the seller or service provider before a transaction is initiated. In key exchange protocols used for example in the Secure Socket Layer (SSL) protocol, the identities of the physical entities are not known to each other before the session is set up. An authentication for these types of situations, where the entities do not know each other, the use of digital signatures and one or more public key infrastructures with trusted CAs is necessary, whereas for pre-established situations several other forms of authentication mechanisms are possible.

In all cases, it is important that the identities are in such a form as to allow the entity to be traced, for example, to settle a dispute. The identities used in pre-established authentication processes are controlled and defined by the establishing procedure and can thus take any form, but in the other more general cases, the identity of an individual should not only be taken from the name, which can be a unique alias, but also from supplementary information which would make it possible physically to reach that person.

\subsection{Data Authentication}

A data authentication process verifies the identity of the origin of data and confirms that the data is intact. An individual can be the sender of the data, such as the originator of an electronic mail communication, but he or she can also be the author, i.e. the originator of the logical content, or the preparer, i.e. the person who physically prepared the data, or the owner, i.e. the person who controls the use of the data. A 
digital signature is the best way to prove the source and the authenticity of the data, since it binds the source to the data in a nonrepudiable way. It is worth pointing out that this use of a digital signature by, or on behalf of, an individual does not imply that the person endorses the content.

The source of data can also be an authority, such as a Certification Authority giving out public key certificates or a Time Stamping Authority (TSA) producing time stamps. In these cases, the digital signature is not made on behalf of a human being but by a physical entity on behalf of an authority. The digital signature is here used in order for anyone to be able to verify, in a non-repudiable way, the identity of the authority that is responsible for the correctness of that data. Note that here the digital signature is actually an endorsement of the content by an authority and not by an individual.

\subsection{Electronic Signature}

An electronic signature authentication verifies not only both the authenticity of signed data and the identity of the individual who electronically signed that data, but also that the data was endorsed by the signer in the same way that a hand-written signature does. Since this is the electronic equivalent of a hand-written signature, the mechanism used should be such that the electronic signature is nonrepudiable. The use of the digital signature mechanism is thus the natural choice.

\section{ELECTRONIC SIGNATURE LAWS}

Money and work can be saved and new business opportunities created if the new information technology could be used more widely in the public and private sectors. In order to speed up this evolution, there is a need to promote the use and acceptability of documents in electronic form. In most legal systems a document is defined as a physical and tangible object, and a signature as needing to be hand-written. A document in electronic form in many countries does not fulfill the legal definition of a document and the fact that an electronic signature 
is not hand-written makes electronic documents sometimes legally unacceptable. There is thus a requirement to adapt most legal systems to the new technology.

Different approaches have been taken. Sweden introduced the concept of electronic document in 1987 in its customs laws [5]. It defined an electronic document as "an electronic document is a recording whose contents and issuer can be verified through a particular technical procedure." In 1995, Utah Digital Signature Act was approved and equates the digital signature to a hand-written signature [6]. Unlike the Swedish approach, this law is very technical, since it strictly binds the law to the digital signature mechanism. Although a law ought to be technically neutral, the Utah state approach has been followed by most other states and nations around the world, as for example the German digital signature law of 1997 [7]. The European Union proposed a directive on electronic signatures in 1998 [2], which in theory is more general but in practice is also digital signature oriented. The proposed implementation of the Government Paperwork Elimination Act in the United States of 1999 [8-9], describes on the other hand a number of different mechanisms. The two latter recognize the terminology problem and use the term electronic signature instead of digital signature. The primary intention of all these laws is to legally permit the use of an electronic equivalent of a hand-written signature, i.e. an electronic signature, in order to promote the use of IT.

The fact that an electronic signature is in digital form has unfortunately led many to use the term digital signature without realizing that this was a well established term used for a mechanism that can be used for an electronic signature, but not exclusively so. This dual use of the term digital signature has caused, and is still causing, lots of confusion. A closer look, for example, at the German Digital Signature law (SigG) [7] reveals that it is actually trying to regulate the use of digital signatures, but only for the purpose of electronic signatures. 


\section{A DISCUSSION OF SOME FORESEEN PROBLEMS AND ISSUES}

In this section, several foreseen problems are discussed. It does not claim to be a comprehensive discussion nor even a discussion of the most critical issues. It rather tries to look at some of the issues not commonly heard in the public debate.

How do we know when a digital signature is used for an electronic signature?

We have seen that digital signatures can be used for three completely different purposes. This leads us to the question: how do we know for which purpose a particular digital signature has been used? This is a very serious problem. Should a digital signature on a document be viewed simply as the source of maybe a forwarded document or should it be considered as an endorsement of the content? This important problem seems to have been ignored in the existing and drafted regulations, where all uses seem to imply an endorsement.

If there is a dispute as to whether a digital signature stands for data authentication or for an electronic signature, one has to find out the context under which the digital signature was created. One way of determining the context is to have a different public key pair for each purpose and for the associated public key certificate to state for which purpose each key pair is intended. This would make it technically clean, but very hard for the users to manage. The user has to possess a number of private keys and associated public key certificates. It will also be hard to prove that the user has not by mistake used the wrong key. An alternative method is to let a user have a single key pair for all types of digital signatures and to include in the signed data an explicit statement of the purpose of the signature. This scheme will probably be more trustworthy, since the signer's action in stating the purpose rather than just engaging one of the set of almost indistinguishable digital signature mechanisms is more likely to avoid mistakes.

Should electronic signatures be limited to human beings ? 
The proposed European electronic signature directive and several other laws that exist, or are under development, have limited their scope to signatures made by natural persons, either as an individual or as a legal person. This is due to the fact that a traditional hand-written signature can by definition only be created by human beings. Before the existence of intelligent IT services, decisions and endorsement were the exclusive property of human beings, but in the new information society era we have to realize that these functions can also be made by intelligent services. Typical examples are the signing of public key certificates and time stamps. These are not signed on behalf of an individual but by a server on behalf of the CA and TSA respectively. The future will probably see more of these types of services, in particular various types of trusted third party services acting on behalf of some trusted authority. Data objects will here be endorsed and signed by servers on behalf of authorities and trusted by society to the extent that the authorities are trusted. As a consequence, within the information society with its intelligent decision making services, it may be that both individuals and certain physical entities will be acting and endorsing data on behalf of a legal person. Most legal systems ought thus to be extended to incorporate this new and more general understanding of an endorsing electronic signature.

\section{For how long should an electronic document and electronic signature be readable?}

In our society some documents are very short-lived, for instance for the duration of the transaction itself, whereas others have to be saved for historical reasons. In the latter case, it is not that easy to permit documentation on media that require specific technical equipment in order to be interpreted. There is a risk that historians will be forced to borrow equipment from museums in order to read historical documents in electronic form.

Those were the extreme cases, but there also exists a number of other situations with requirements for readability over a time range of one month, one year, 10 years, 30 years etc. Not only is it a matter of which media, it is also a matter of providing protection against security breaches. The length of cryptographic keys does not, for 
example, have to be too long for short-lived digital signatures. Medium or long term documents might on the other hand require electronic signatures to be regularly re-signed by some trusted authority [10]. Some nations have started work and tests in this area, but it is not expected that an international consensus can be reached in the near future on the best approach. Further work and research on this difficult and tricky problem are recommended

\section{Why are most signature laws not technically neutral ? \\ Why do they mention digital signatures? Why not just define a document in electronic form?}

The main purpose of an electronic signature law is to promote the acceptability, and thereby the use, of documents in electronic form. One then asks the question: why has most legislation adopted the approach of regulating a particular type of technical mechanism in order to achieve this goal?

There are probably several reasons. One is that in some countries the legal system has to adapt not only the definition of a document but also the definition of a signature. Another is that all other mechanisms are technically much weaker than digital signatures. A third might be due to the fact that many proposals have been drafted by technicians.

If the extension of a document definition to include its electronic form is the only legal modification necessary, then the approach taken in the Swedish customs law mentioned above would be sufficient [5]. If the legal concept of a signature has to be extended, then electronic signatures have to be included. This is what the EU directive [2] and the US proposal [8] have done, although it is clear that the text of the former has been written with digital signatures in mind.

In principle, an electronic signature can be made by a variety of mechanisms. The quality and level of trust in the signature will however greatly depend on the mechanism. It is due to this wish to provide a state of the art solution that the digital signature has received a particular status, since it is arguably by far the best and secure mechanism known today. No other mechanism is comparable in 
strength, with the possible exception of the involvement of additional third party services, like notary services.

Most of those regulating the electronic signature want to provide a very secure and trustworthy signature and have tried to provide a detailed description of requirements that would to a large extent diminish certain threats and weaknesses. However, complete security can never be achieved, and some weaknesses have been completely ignored. The trust in the authenticity of a particular electronic signature always has to rely on a judgment based on a number of factors.

The criticism of specifications with a high level of protection against certain weaknesses without a guarantee of the whole electronic signature process should not be ignored, since technical and economic burdens might delay rather than promote the evolution. Since it is hard to foresee all weaknesses that can be exploited, it might instead be better to improve only gradually the quality and requirements of this new technology, based on the experiences gained in the real world. The approach that best promotes the use of IT in the public and private sectors is hard to judge, but in the laws of those countries where legislation has already been introduced, or is under discussion, a reflection of the nation's culture can be traced. The most important and probably the only necessary statement in any signature law is that an electronic signature should not be discarded on the sole ground that it is in electronic form.

\section{Can anything be done about the inter-cultural problems ?}

When the public key infrastructure is used to provide individuals with a form of electronic ID-cards, like a passport, the names have to be in the person's native language. This may cause some inter-cultural difficulties if this form of identity is used for instance in electronic commerce. This is an illustration of the fact that cyberspace, which does not recognize any physical boundaries, is instead culturally divided. Another example of a culture difference is the way in which confidence between business partners is established. Some of these cultural segmentation issues are fairly technical, such as languages 
and character sets, whereas others are more behaviour-oriented in nature. The discussion here will be restricted to the former, since only these might be overcome by technical means.

The use of different languages and, in particular, character sets can cause problems when identifying human beings, for example, in electronic commerce. If a user has an electronic ID card of some type, it will probably be based on some public key authentication scheme. An X.509 public key certificate allows the name of the individual associated with that key pair to be specified with any character set of the Universal Multiple-Octet Coded Character Set [11]. There is no problem with the interchange of such public key certificates. The problem arises if a service provider, for example, in North America cannot display and interpret correctly the name of an individual, for example, from Greece or Asia. Can the provider really identify the customer in such a way that he or she can be reached in case of a dispute? Can the provider even display the identity at all and does it make any sense to him or her?

A possible solution for the technical problem raised above would be to have an internationally recognized agreement, for example, in the form of an international standard, determining which attributes should be included in a public key certificate for ID purposes, how they should be specified, and what requirements must be met within an entity authentication application in order to make electronic ID certificates globally useable.

\section{SUMMARY}

The public debate arising from the introduction of laws about signatures in electronic form has prompted this paper. It attempts to clarify the relationships between authentication, digital signatures and electronic signatures. Issues that have not commonly been seen in the debate have been identified, analyzed and discussed. How widespread and important the use of digital signatures and in particular digital signatures for electronic signatures will become, and how fast they will be taken up in society, it is hard to judge, but most likely this will 
be determined by an evolutionary development based on practical experience supported by legislation. The discussion and proposals made in this paper are intended to contribute in a positive way to wider understanding and acceptability of both digital signatures and electronic signatures for successful and expeditious transition to the new and exiting information society already on our doorstep.

\section{REFERENCES}

[1] ISO 7498-2 (1988) Information Processing Systems - Open Systems Interconnection Reference Model - Part 2: Security Architecture

[2] COM(1998)297 final: Proposal for a European Parliament and Council Directive on a common framework for electronic signatures (May 13,1998)

[3] RFC 2459 (1999) Internet X.509 Public Key Infrastructure Certificate and CRL Profile

[4] ITU-T Recommendation X.509, Information Technology - Open Systems Interconnection - The Directory: Authentication Framework (1996)

[5] (Section 11 a) in the Swedish Customs Act (1987:1065)

[6] The Utah Digital Signature Act (1995)

[7] The German Digital Signature Act - Signaturgesetz - SigG (1997)

[8] The proposed Implementation of the Government Paperwork Elimination Act http://www.access.gpo.gov/su_docs/aces/fr-cont.html (March 5, 1999)

[9] Government Paperwork Elimination Act (GPEA) Pub. L. 105-277, Title XVII (October 21, 1998)

[10] H. Nilsson and D. Pinkas, Validation of Electronic Signatures, http://www.openmaster.com/whitepaper/es_validation.pdf

[11] ISO/IEC 10646-1 (1993) Information Technology - Universal Multiple-Octet Coded Character Set (UCS) - Part 1: Architecture and Basic Multilingual Plane 